\title{
Influence of perpendicular magnetic anisotropy on closure domains studied with $\mathrm{x}$-ray resonant magnetic scattering
}

\author{
E. Dudzik, S. S. Dhesi, ${ }^{*}$ H. A. Dürr, ${ }^{\dagger}$ S. P. Collins, M. D. Roper, and G. van der Laan \\ Magnetic Spectroscopy Group, Daresbury Laboratory, Warrington WA4 4AD, United Kingdom \\ K. Chesnel, M. Belakhovsky, A. Marty, and Y. Samson \\ Département de Recherche Fondamentale sur la Matière Condenseé, Service de Physique des Matériaux et des Microstructures, \\ CEA Grenoble, 17 rue des Martyrs, 38054 Grenoble, France
}

(Received 15 February 2000)

\begin{abstract}
FePd thin-film samples with perpendicular magnetic anisotropy (PMA) have been studied with x-ray resonant magnetic scattering, both at the Fe and at the $\mathrm{Pd} L_{3}$ edges. In these samples the competition between PMA and shape anisotropy leads to the formation of highly ordered striped domain patterns with a magnetization component perpendicular to the film plane. These striped domains give rise to magnetic satellite peaks in the diffraction pattern. Magnetic diffraction rod scans of these satellites were analyzed to obtain information about the magnetic depth profile of the films. It was found that flux closure occurs in samples with a low to medium PMA, while a high PMA impedes the formation of closure domains. Data analysis gives a depth of the closure domains extending to $85 \AA$, with approximately half the magnetic moment aligned in plane.
\end{abstract}

\section{INTRODUCTION}

Magnetic media with perpendicular magnetic anisotropy (PMA) are currently of great interest as one of the most promising materials for increasing magnetic data storage densities. ${ }^{1}$ It is thus both technologically and fundamentally important to study the exact magnetic configurations that PMA will produce in small magnetic structures. The samples studied in this experiment were FePd thin films with varying degrees of PMA, which were grown by codepositing Fe and $\mathrm{Pd}$ at elevated temperatures. Depending on the exact growth conditions, the resulting films are more or less chemically ordered, with $\mathrm{Fe}$ and $\mathrm{Pd}$ occupying alternating layers in a tetragonally distorted fcc lattice. ${ }^{2-5}$ This chemical ordering leads to the PMA, so that the anisotropy increases with the degree of order. In moderately well-ordered films, the competition between the PMA and the thin-film shape anisotropy leads to the appearance of well-ordered striped domains with a magnetization profile $\uparrow \downarrow \uparrow \downarrow \uparrow \downarrow \uparrow$ perpendicular to the film plane. Since this magnetic structure is characterized by magnetic-flux lines partly outside the film, flux closure should be energetically favorable, which leads to closure domains at the film surfaces. ${ }^{6}$ The resulting domain structure consists of closed magnetic loops with alternating clockwise and counterclockwise orientation, i.e., $\uparrow \rightarrow \downarrow \leftarrow \uparrow \rightarrow \downarrow \leftarrow \uparrow$. The presence of these closure domains has already been demonstrated by Dürr et l $^{7}{ }^{7}$ using circularly polarized light to detect the circular domain structure. In films with a strong PMA there are still flux lines outside the film, but the formation of closure domains with an in-plane magnetization becomes energetically unfavorable, as there is a pronounced easy axis in the vertical direction.

Because conventional magnetic imaging techniques rely on the detection of stray magnetic fields outside the sample, closure domains are difficult to observe. In this paper X-ray resonant magnetic scattering (XRMS) is used for detailed magnetic depth profiling of FePd thin films. XRMS makes use of the element specific resonant enhancement of the magnetic scattering amplitude, which occurs when the scattering process involves a strong electric multipole transition from an atomic core level to unoccupied states above the Fermi level. The magnetic sensitivity of the scattering cross section is due to the spin polarization of the unoccupied valence bands. In the case of the $3 d$ transition metals, large enhancements occur at the $L_{2,3}$ absorption edges. ${ }^{8}$ As a consequence, the method is only suitable for studying structures with a period of at least half the $\mathrm{x}$-ray wavelength $(\lambda \sim 15$ $\AA$ ). While this excludes Bragg diffraction peaks from crystalline materials, it enables the study of magnetic multilayers and magnetic domain structures. ${ }^{\text {? }}$

Here we have studied a series of samples with increasing PMA. Evidence for the existence of closure domains in samples with a low to medium PMA can be found in magnetic diffraction rod scans as well as the dichroic asymmetry ratio of the magnetic satellite peaks. Rod scans from a highPMA sample show no sign of closure domains. The method of data evaluation is briefly outlined and structural and magnetic parameters obtained by fitting both the magnetic rod scans and the dichroic asymmetry ratio are given.

\section{EXPERIMENT}

\section{A. Samples}

The samples were grown at the Commissariat à l'Energie Atomique (CEA) in Grenoble by depositing approximately $400 \AA$ of $\mathrm{Fe}$ and $\mathrm{Pd}$ under UHV conditions on an $\mathrm{MgO}$ substrate. The FePd layer was capped with $20 \AA$ of Pd to prevent oxidation. Growth conditions were varied to produce a range of different anisotropies, and are listed in Table I together with sample properties. The samples used in this experiment had anisotropies $Q$ of $0.4,0.8$, and 1.6, with $Q$ $=K_{u} / 2 \pi M_{s}^{2}$, where $M_{s}$ is the saturation magnetization per 
TABLE I. CEA reference number, sample structure and layer thicknesses (substrate/buffers/FePd film/ capping layer), FePd layer growth process and growth temperature $T_{\mathrm{G}}$, magnetic anisotropy $Q$ $=K_{u} / 2 \pi M_{s}^{2}$, the stripe period $\tau$ determined by MFM, and the in-plane correlation length $L_{\mathrm{C}}$ (determined by $\mathrm{XRMS}$ ) for the three samples studied in this work.

\begin{tabular}{|c|c|c|c|c|c|c|c|}
\hline & Ref. & Structure & Growth & $T_{\mathrm{G}}(\mathrm{C})$ & $Q$ & $\tau(\mu \mathrm{m})$ & $L_{\mathrm{C}}(\mu \mathrm{m})$ \\
\hline Sample I & 525 & $\begin{array}{c}\mathrm{MgO} / \mathrm{Cr}(60 \AA) / \operatorname{Pd}(720 \AA) \\
/ \operatorname{FePd}(430 \AA) / \operatorname{Pd}(20 \AA)\end{array}$ & layer-by-layer & RT & 0.4 & 0.09 & 1.6 \\
\hline Sample II & 515 & $\mathrm{MgO} / \mathrm{FePd}(400 \AA) / \mathrm{Pd}(20 \AA)$ & codeposited & 220 & 0.8 & 0.09 & 0.63 \\
\hline Sample III & 599 & $\begin{array}{c}\mathrm{MgO} / \mathrm{Cr}(30 \AA) / \mathrm{Pd}(600 \AA) \\
/ \operatorname{FePd}(400 \AA) / \operatorname{Pd}(20 \AA)\end{array}$ & codeposited & 370 & 1.6 & 0.1 & 0.59 \\
\hline
\end{tabular}

volume unit and $K_{u}$ the perpendicular anisotropy constant. Throughout the paper these samples are referred to as I, II, and III in order of increasing anisotropy. In the two samples with the lower quality factors the striped PMA domains form spontaneously, because there is a comparatively large inplane component of the magnetization due to the shape anisotropy. This in-plane moment directed along the length of the domains leads to high costs in exchange energy for any sharp curvature in the domains. In sample III with a high PMA a striped domain pattern can only be generated by applying an external in-plane field at the end of the growth process. All samples were characterized by magnetic force microscopy (MFM); the width of the stripes was generally found to be $\sim 0.09 \mu \mathrm{m}$.

\section{B. X-ray magnetic scattering}

Experiments were carried out at the European Synchrotron Radiation Facility (ESRF) in Grenoble on the helical undulator beamlines ID12B and ID12A, and on stations 5U1 and 1.1 of the Daresbury Synchrotron Radiation Source (SRS), using both linearly and circularly polarized light. A Daresbury two-circle diffractometer vacuum chamber ${ }^{10}$ was used throughout. Experiments were performed in two different geometries, where the detector (a photodiode) could be scanned either within or perpendicular to the scattering plane. In the first geometry (labeled A), "conventional" $\theta-2 \theta$ reflectivity curves and rocking scans could be obtained. We also measured diffraction rod scans of magnetic satellite peaks. In the second geometry, B, a series of transverse scans could be measured for a range of fixed angles of incidence $\theta$.

\section{DATA ANALYSIS}

\section{A. Parametrization of the sample magnetization}

Two aspects of the magnetization profile have to be modeled. One is the in-plane periodic profile from the PMA striped domains, closure domains, and domain walls, the other is the perpendicular magnetization profile, i.e., the magnetic layer thickness, interface roughness, depth of the closure domains, etc. The periodic magnetization profile was approximated by the first two terms of a Fourier series (only odd terms are needed for symmetry reasons). Assuming that the magnetization profile with a modulation vector $\tau$ has a period of $2 \pi / \tau$ along the $x$ direction (this is the case in geometry A), this results in

$$
\begin{gathered}
M_{x}=M_{x}^{1} \cos (\tau x)+M_{x}^{3} \cos (3 \tau x), \\
M_{y}=0, \\
M_{z}=M_{z}^{1} \sin (\tau x)+M_{z}^{3} \sin (3 \tau x),
\end{gathered}
$$

where the components $M_{i}^{1}$ and $M_{i}^{3}$ have to be determined by fitting. The $z$ component of the magnetization relates to the PMA domains, the $x$ component to the closure domains. In samples with low or medium PMA there is a small in-plane contribution from the shape anisotropy in the $y$ direction, but because this moment is almost constant, it contributes little to the diffraction peaks and has therefore been neglected in the present model.

Perpendicular to the film plane the magnetic FePd layer is sandwiched between two nonmagnetic Pd layers. The magnetic profile in this direction is assumed to consist of a top layer containing both closure domains and PMA domains, a bulk layer of PMA domains, and a second closure domain layer at the bottom of the film, where the magnetization in the closure domains has the opposite sign. Figure 1 shows the relative position of PMA and closure domains, as well as an example of the magnetic depth profiles used to analyze the data. The interfaces between the magnetic FePd layer and the capping/buffer layers were Gaussian broadened to simulate roughness, as were the interfaces between the closure layers and the bulk. This gives a total of four fit parameters: the total thickness of the FePd layer $d$, the thickness of the closure domain layer $d_{c}$, and the two magnetic interface roughnesses.

\section{B. Calculated diffracted intensity}

The basis of the data fitting is the resonant magnetic scattering amplitude $f_{n}$ for electric dipole transitions which can be found elsewhere. ${ }^{11-13}$ The scattered intensity is proportional to the modulus square of the scattering amplitude times a phase factor, summed over the site $\mathbf{r}_{n}$ of each resonant atom,

$$
I \propto\left|\sum_{n} e^{i \mathbf{q} \cdot \mathbf{r}_{n}} f_{n}\right|^{2},
$$

where $\mathbf{q}=\mathbf{k}^{\prime}-\mathbf{k}$ is the momentum transfer between incoming and scattered beam. The scattering amplitude $f_{n}$ is 

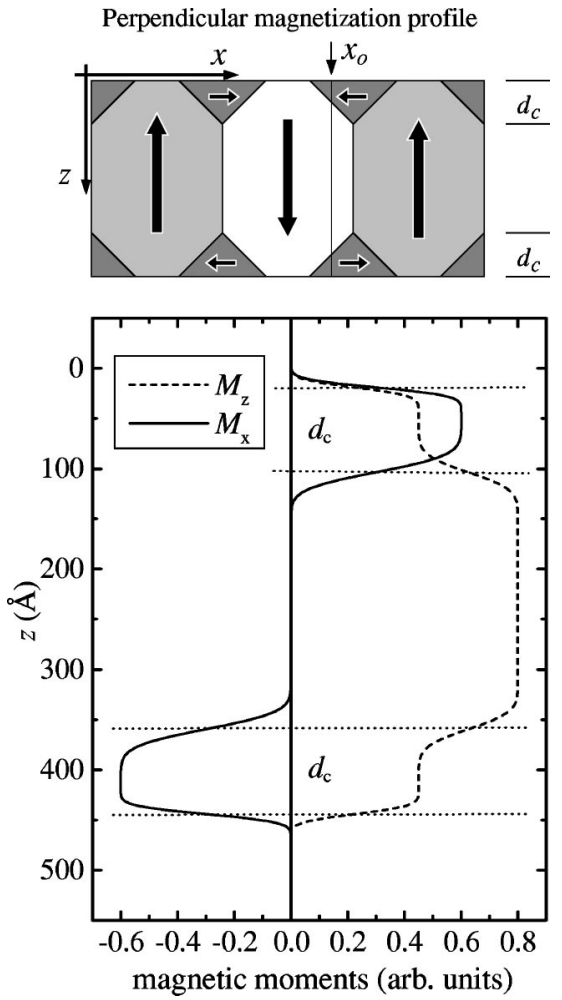

FIG. 1. Top: model of closure domains with an in-plane magnetization in a thin film with striped PMA domains. Bottom: example of the magnetization profile for the $x$ (solid line) and $z$ magnetization (dashed line) at point $x_{0}$ used for data analysis. The $x$ magnetization changes sign in the top and bottom closure domains. Transitions between the closure and bulk layers are broadened by Gaussians.

$$
f_{n}=\hat{\mathbf{e}}_{f} \cdot \hat{\mathbf{e}}_{i} F_{n}^{(0)}-\mathrm{i}\left(\hat{\mathbf{e}}_{f} \times \hat{\mathbf{e}}_{i}\right) \cdot \hat{\mathbf{M}}_{n} F_{n}^{(1)}+\left(\hat{\mathbf{e}}_{f} \cdot \hat{\mathbf{M}}_{n}\right)\left(\hat{\mathbf{e}}_{i} \cdot \hat{\mathbf{M}}_{n}\right) F_{n}^{(2)},
$$

where $\hat{\mathbf{e}}_{i}$ and $\hat{\mathbf{e}}_{f}$ are the unit polarization vectors of the incident and the scattered $\mathrm{x}$ rays, respectively, and $\hat{\mathbf{M}}_{n}$ is the unit magnetization vector at site $n$ in the sample. The complex factors $F_{n}$ describe the atomic resonant excitation and decay processes. The first term of $f_{n}$ is independent of the magnetization and contributes to the charge Bragg peak. The linear and quadratic terms in $\hat{\mathbf{M}}$ produce the first-order and higherorder magnetic satellites.

The dipole operator can be written as a $(2 \times 2)$ matrix by using polarization vectors perpendicular $(\sigma)$ and parallel $(\pi)$ to the scattering plane. For the first-order magnetic term of the scattering amplitude the result is

$$
\left(\hat{\mathbf{e}}_{f} \times \hat{\mathbf{e}}_{i}\right) \cdot \hat{\mathbf{M}}_{n}=\left(\begin{array}{cc}
0 & \hat{\mathbf{k}} \\
-\hat{\mathbf{k}}^{\prime} & \left(\hat{\mathbf{k}}^{\prime} \times \hat{\mathbf{k}}\right)
\end{array}\right) \hat{\mathbf{M}}_{n} .
$$

The relative size of the contributions from the three possible scattering channels $(\sigma \rightarrow \pi, \pi \rightarrow \sigma$, and $\pi \rightarrow \pi)$ depends on the polarization of the incident beam. If the beam is purely $\sigma$ polarized, only $\sigma \rightarrow \pi$ scattering can occur. If the beam is circularly polarized, the polarization vector is $\mathbf{p}^{ \pm}$ $=\hat{\mathbf{e}}_{\sigma} \pm i \hat{\mathbf{e}}_{\pi}$, and all three scattering channels are possible. Their contributions must be added up taking the phase shift between the $\sigma$ and $\pi$ components of the incident beam into account. This phase shift can lead to interference between the $\sigma \rightarrow \pi$ and $\pi \rightarrow \pi$ scattering channels when there is a periodic magnetic moment perpendicular to the scattering plane, which appears as circular dichroism in the magnetic scattering.

The sine and cosine terms of the magnetization profile [Eq. (1)] can be written as complex exponents and incorporated into Eq. (3). The resulting (rather lengthy) expression can be sorted into terms corresponding to various orders of diffraction.

It is instructive to work out the magnetic scattering contribution for the $\sigma \rightarrow \pi$ channel in geometry A as an example, neglecting interference between different orders. For the sake of clarity only the first-order term of the Fourier series for the magnetization is used. For the $\sigma \rightarrow \pi$ channel in Eq. (4) we obtain with Eq. (1)

$$
\hat{\mathbf{k}}^{\prime} \cdot \hat{\mathbf{M}}_{n}^{1}=k_{x}^{\prime} M_{x}^{1}\left(\frac{e^{i \tau x}+e^{-i \tau x}}{2}\right)-i k_{z}^{\prime} M_{z}^{1}\left(\frac{e^{i \tau x}-e^{-i \tau x}}{2}\right) .
$$

To calculate the intensity, the summation over $x$ and $y$ is carried out (the $y$ term drops out since the momentum transfer in the $y$ direction is zero),

$$
I \propto\left|\sum_{n} e^{i q_{z} z} \delta(q \pm \tau)\left(k_{x}^{\prime} M_{x n}^{1} \mp i k_{z}^{\prime} M_{z n}^{1}\right) F_{n}^{(1)}\right|^{2} .
$$

The periodicity of the magnetization in the $x$ direction leads to magnetic satellites at positions of $q_{x}= \pm \tau$ in reciprocal space. In an analogous way, including the higher terms in the Fourier series leads to higher-order magnetic satellites. The first-order magnetic scattering term produces odd order satellites, the second-order magnetic scattering structure factor contributes to the even order satellites. In the experiment only the first- and second-order satellite peaks could be observed. It should be noted that, since we cannot measure absolute scattered intensities and do not know the coefficients $F$, we can only determine the ratios of the Fourier coefficients $M_{n}^{i}$.

To obtain the magnetic rod scans, the intensity of the satellite peak at each angle of incidence $\theta$ is calculated by carrying out the summation over $z$, starting at $z=0$ at the top of the sample, and increasing $z$ until the $\mathrm{Pd}$ buffer layer is reached where the magnetization is zero. For every $z$ the magnetization is calculated using the fit parameters for the perpendicular magnetic profile, and the Fourier components of the periodic profile. The absorption in the material is simulated by multiplying $f_{n}$ with $\exp (-z / l \sin \theta)$, where $l$ corresponds to the penetration depth.

\section{RESULTS AND DISCUSSION}

\section{A. Magnetic satellites}

Figure 2 shows diffraction scans taken at the Fe $L_{3}$ absorption edge in geometries A and B with circularly polarized light. In geometry A the striped domains were perpendicular to the scattering plane and the sample was rocked while the detector was kept at a constant angle (see insets in Fig. 2). In geometry B, the stripes were parallel to the scat- 


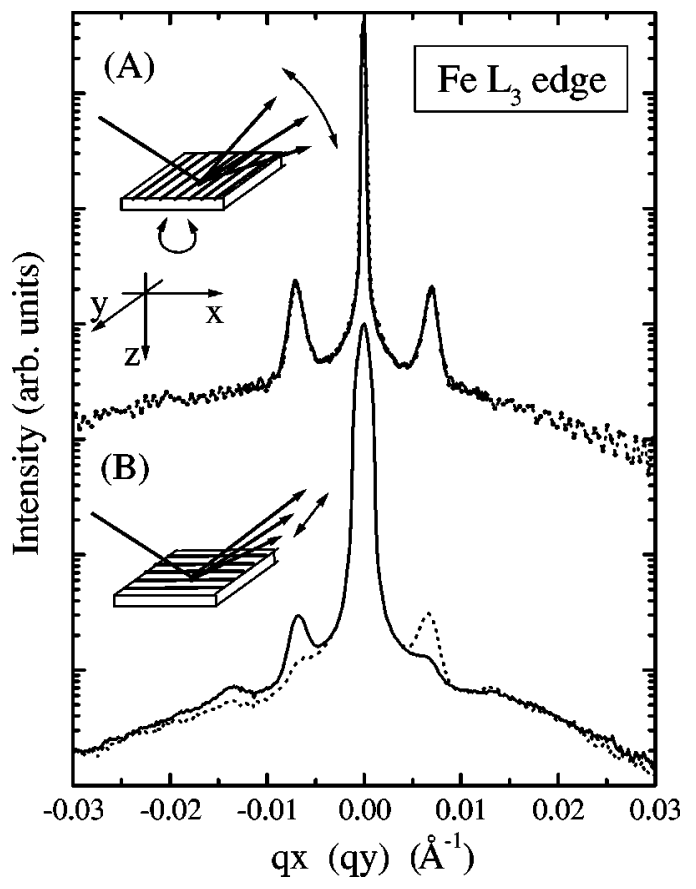

FIG. 2. Magnetic satellite peaks observed around the specular peak for sample II in scattering geometries A (top) and B (bottom) at $709 \mathrm{eV}$ photon energy, with left (dotted line) and right (solid line) circularly polarized light. X-ray circular dichroism only occurs in geometry B. The coordinate frame is fixed to the scattering plane and is used throughout the paper.

tering plane, and the detector was scanned horizontally across the diffraction pattern. The different widths of the specular peak in the two geometries are due to the beam profile. In both geometries we see satellite peaks, offset from the central specular peak by $\sim 0.007 \AA^{-1}$. This corresponds to a real space period of $0.09 \mu \mathrm{m}$, which is in good agreement with the MFM data for the striped domains. The satellites are only observable for energies within a few $\mathrm{eV}$ of the absorption edge, which shows that they are magnetic in origin. The satellite signal was maximized by scanning the energy across the absorption edge while keeping the reciprocal space position fixed at the first-order satellite to find the maximum signal.

In geometry A the second-order peaks are too weak to be observable, and no higher orders were found. There is no difference in the scattering of negative and positive circularly polarized light. In geometry B, on the other hand, secondorder peaks are visible, and the magnetic satellites show a strong circular dichroism with opposite sign on either side of the specular peak. This dichroism is an indication of the presence of a chiral domain pattern. ${ }^{7}$

Figure 3 compares the first-order magnetic diffraction peaks measured in geometry $\mathrm{A}$ at the $\mathrm{Fe} L_{3}$ edge of samples I and II. The lines are Gaussians fitted to the data. As explained above, sample I, which has a lower degree of chemical order (and thus weaker PMA) is expected to have a better ordered striped domain pattern. This can be seen to be the case, the magnetic diffraction peak for sample I is considerably narrower than that of sample II, with correlation lengths of 1.6 and $0.63 \mu \mathrm{m}$, respectively. In sample III the in-plane correlation length for the striped domains is $0.59 \mu \mathrm{m}$, slightly shorter than in sample II.

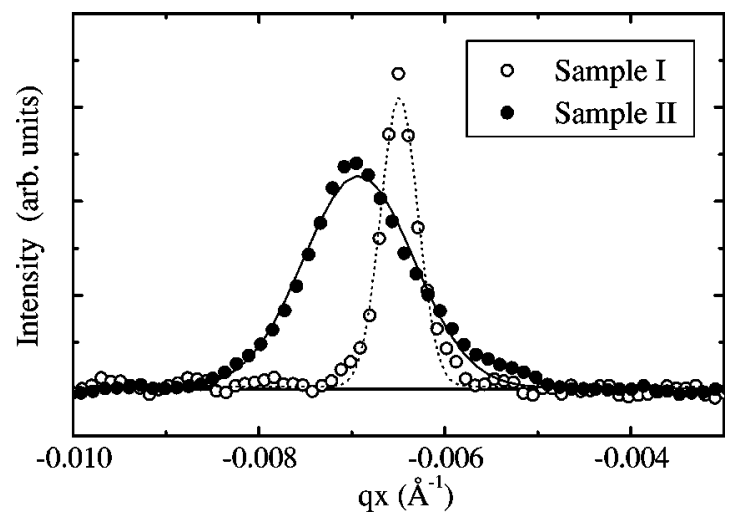

FIG. 3. Width of the magnetic satellite peaks measured in geometry A for samples I and II. The diffuse background has been subtracted and Gaussian functions fitted (solid/dashed lines) for both data sets to obtain the peak width. The striped domains have a much better magnetic order in the low-PMA sample I.

We also observed magnetic satellites at the $\mathrm{Pd} L_{3}$ edge (Fig. 4), although they are much weaker relative to the specular peak than at the Fe edge. The magnetic scattering signal is reduced by a factor of 10 , because the induced magnetic moment of the Pd atoms $\left(0.65 \mu_{B}\right)$ is only a third of that of the $\mathrm{Fe}\left(2.2 \mu_{B}\right){ }^{3}$ This means that the satellites are just visible above the diffuse background in Fig. 4 in geometry A; no satellites were observed in geometry B.

\section{B. Rod scans}

Specular reflectivity and magnetic rod scans were measured in geometry A at both absorption edges. At the $\mathrm{Pd}$ edge the magnetic rod scan had to be measured by scanning across the magnetic peak for a large range of angles of incidence $\theta$. The diffuse background was then subtracted and the peak intensity integrated at each point. Figure 5 shows the resulting magnetic rod scan for sample I (a), together with a specular reflectivity scan (b) at the $\operatorname{Pd} L_{3}$ edge. The insets show the corresponding Fourier transforms of the rod scans. The specular rod shows interference fringes which originate from various layers within the sample-not only the $420 \AA$ FePd layer, but also a component corresponding to the overall Pd layer (buffer layer + FePd + capping layer), and some

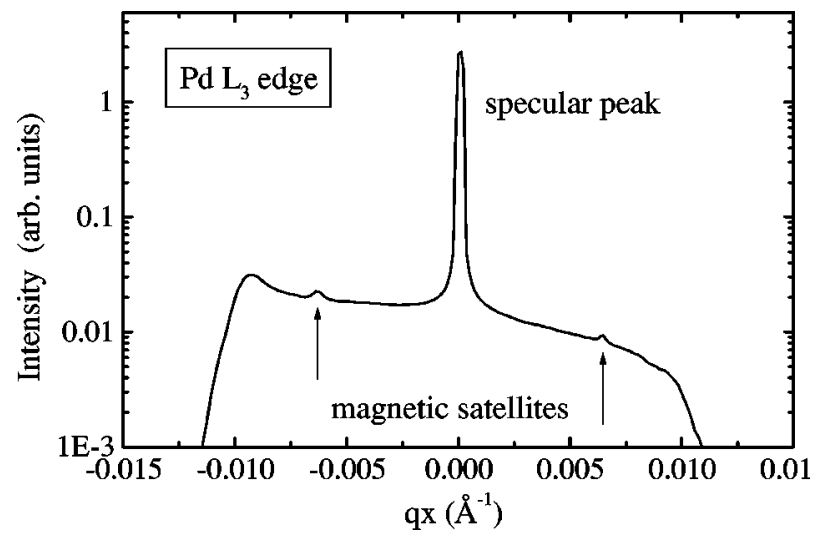

FIG. 4. Magnetic satellite peaks observed around the specular peak in geometry $\mathrm{A}$ at the $\mathrm{Pd} L_{3}$ edge for sample I. 


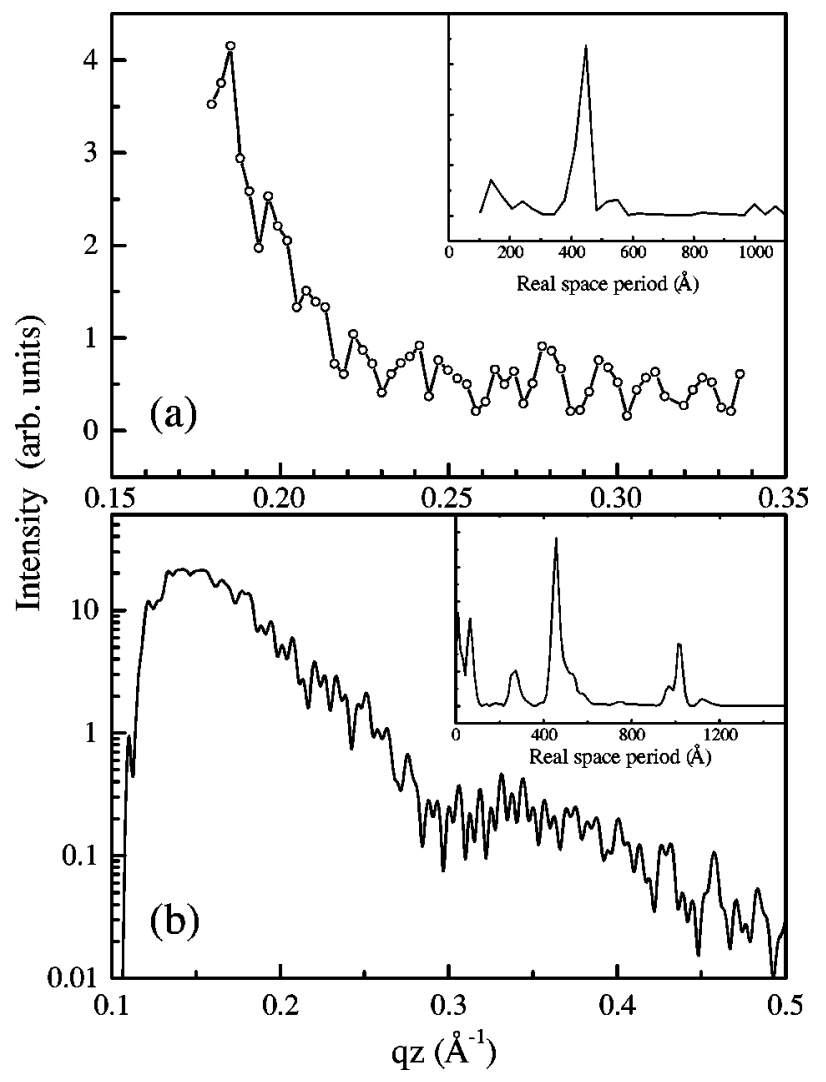

FIG. 5. Rod scans of (a) the magnetic satellite peak for $q_{x}$ $=0.0069 \AA^{-1}$ at the Pd $L_{3}$ edge, and of (b) the specular reflectivity of sample I. The insets show the corresponding Fourier transform.

contributions from smaller periods. The magnetic rod on the other hand is clearly dominated by the interference fringes from the magnetic FePd layer, with only a single contribution in the Fourier transform, since the nonmagnetic layers are "invisible" to the magnetic scattering. This rod therefore contains only information of the magnetic FePd film.

At the $\mathrm{Fe} L_{3}$ edge the difference between the specular and the first-order magnetic rod is not so striking, since the penetration depth at $710 \mathrm{eV}$ is an order of magnitude shorter than at $3174 \mathrm{eV}$. However, the shorter penetration depth leads to a higher surface sensitivity of the signal, which makes it easier to observe the effects of the closure domains. Figure 6 shows the magnetic diffraction rods from sample II with medium and sample III with high PMA. Both rods show an initial increase due to total reflection at low angles of incidence $\theta$. The rod scan from sample III shows no further features beyond an exponential decay. Sample II, on the other hand, shows a distinct shoulder and some minor features at higher $q_{z}$ in its magnetic rod scan.

Figure 7 shows the sample II rod scan together with several magnetic rods which were calculated following the method outlined in Sec. II. The parameters in the calculation were the relative $x$ and $z$ magnetization in the closure domain layer, the $z$ magnetization of the bulk layer, the respective layer thicknesses and interface roughnesses, and the penetration depth of the $\mathrm{x}$ rays. The rod profile at the bottom (solid line) is calculated for an FePd layer without closure domains, so that there is a uniform magnetic moment in the $z$ direction throughout the film. It can be seen that the rod profile, after

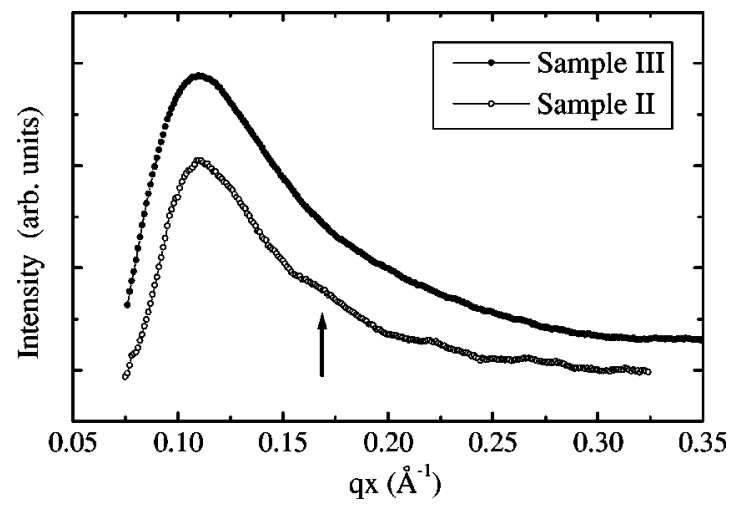

FIG. 6. Rod scans of the magnetic satellite peak taken at the Fe $L_{3}$ edge with a fixed momentum transfer for samples III (full circles) and II (open circles). The arrow indicates a shoulder in the sample II rod scan.

the initial rise, is a smooth decay, modulated by the Kiessig fringes from the FePd layer. When a closure domain layer with a nonzero periodic magnetic moment in the $x$ direction is introduced, a shoulder appears in the rod profile, with a separation between the total reflectivity peak and the shoulder that depends on the depth of the closure layer. Comparison with the experimental rod profile suggests a closure domain depth of $\sim 90 \AA$. The assumption that the shoulder is due to the closure domains is further supported by the fact that the high-anisotropy sample rod scan shows only a smooth decay without additional features.

The fits discussed in the following were all performed on data from samples I and II with low and medium anisotropy, respectively. In the high-anisotropy sample III there is some diffuse scattering around the specular peak that makes it difficult to separate properly the magnetic contribution. The magnetic rod fits at the Fe and $\mathrm{Pd} L_{3}$ edges are shown in Fig. 8 for sample I. The fact that calculated and experimental data deviate at higher $q_{z}$ values, might be to some degree due to intensity changes related to the changing size of the footprint of the beam on the sample. Another problem is the diffuse scattering background due to structural roughness in the $\mathrm{Fe}$ edge data, which contributes to the overall signal. Our fitting method cannot account for this contribution, since it only

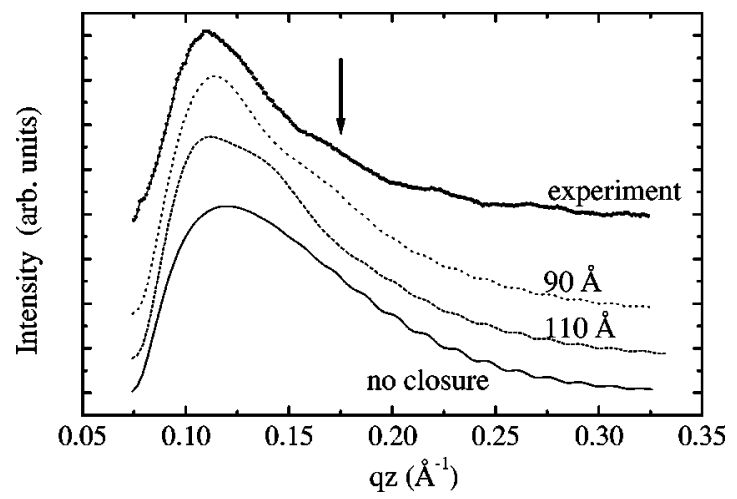

FIG. 7. Influence of closure domain depth on the magnetic rod profile. Solid line: no closure domains, dashed line: closure depth $110 \AA$, dotted line: closure depth $90 \AA$, top line: experiment (sample II). The closure domain layer gives rise to the shoulder (arrow) in the rod profiles. 


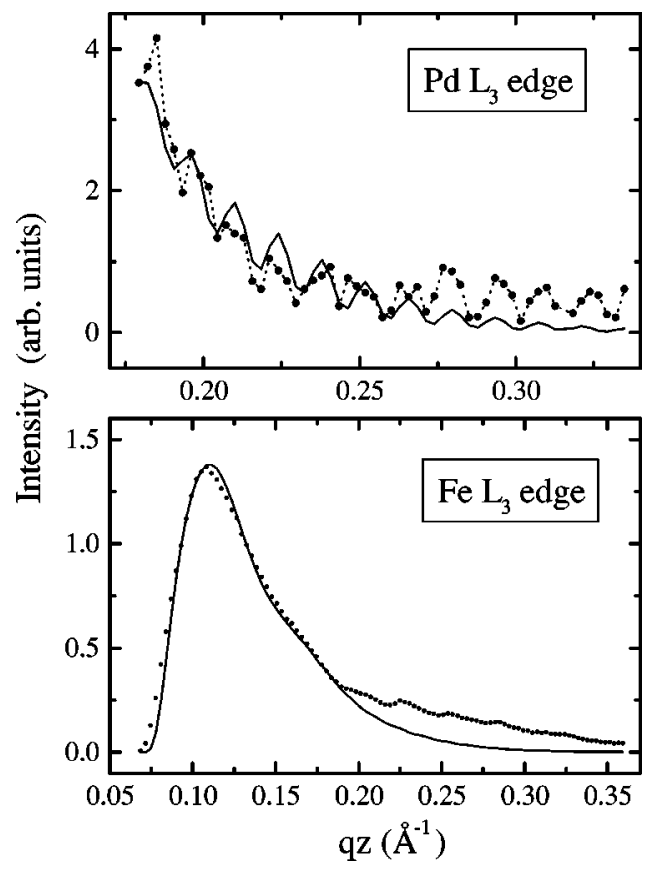

FIG. 8. Fitted magnetic rod scans at the Fe and $\mathrm{Pd} L_{3}$ edges (sample I).

calculates the magnetic scattering. In fitting the data, we therefore concentrated on the first half of the magnetic diffraction rod. It can be seen that the agreement here is reasonably good, both at the Fe and at the Pd edge, although the model of the magnetic depth profile is rather simplified, ignoring for example the effects of domain walls.

These fits can now be compared to results obtained from the angle dependence of the circular dichroism of the magnetic satellites in geometry B using the method described by Dürr et al. ${ }^{7}$ Figure 9 shows the dichroic asymmetry ratio of sample II measured in geometry B for various angles $\theta$. The lines are fits using the same magnetic and structural parameters and essentially the same fit program as for the rod scans (allowing for the different geometry and the interference between $\sigma \rightarrow \pi$ and $\pi \rightarrow \pi$ scattering that occurs here). The fit in Fig. 9 corresponds to a closure domain depth of $83 \AA$, and is extremely sensitive to the relative magnetic moments in

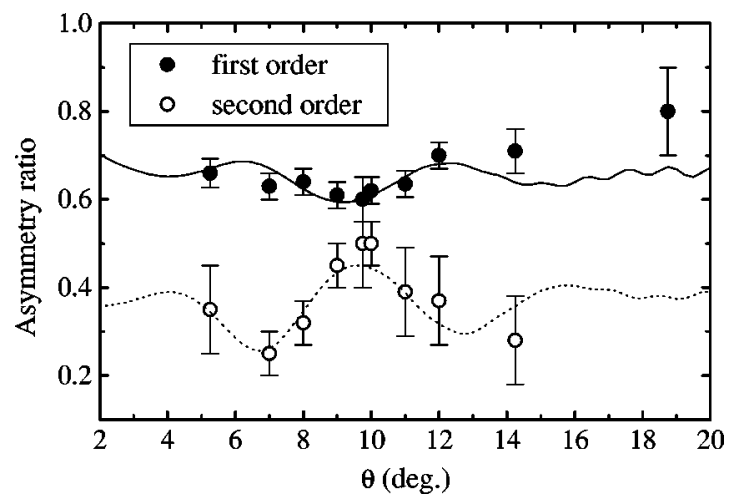

FIG. 9. Asymmetry ratio, $\left(I_{+}-I_{-}\right) /\left(I_{+}+I_{-}\right)$, of the dichroism signal for sample II plotted against angle of incidence $\theta$. This information can be used to obtain a perpendicular magnetic profile of the sample. Dotted and solid lines are fits to the theoretical model.
TABLE II. Comparison of the structural and magnetic fit parameters for sample II obtained from rod scans and the angle dependent dichroic asymmetry ratio. $d_{c}$ is the thickness of the closure domain layer in $\AA, r_{1}$ and $r_{2}$ give the FWHM Gaussian interface roughness at the top and bottom of the FePd layer, and between closure domain and bulk layer, respectively. $M_{z b}^{1}$ is the first Fourier coefficient of the $z$ magnetization in the bulk, and $M_{z c}^{1}$ and $M_{x c}^{1}$ are the first Fourier coefficients of the $z$ and $x$-magnetization in the closure layer. These coefficients are given as relative values of the moments, not as the absolute values. Not listed are the fit parameters for the x-ray penetration depth and film thickness.

\begin{tabular}{lcccccc}
\hline \hline & $d_{c}$ & $r_{1}$ & $r_{2}$ & $M_{z b}^{1}$ & $M_{z c}^{1}$ & $M_{x c}^{1}$ \\
\hline Rod fits & 89 & 9.7 & 20 & 1.0 & 0.45 & 0.6 \\
Asymmetry ratio & 83 & 8 & 18 & 1.0 & 0.5 & 0.55 \\
\hline \hline
\end{tabular}

the bulk and the closure domain layer. The results of this fit can be compared to those found by fitting the magnetic rod profiles. Table II shows the parameters obtained by both methods for sample II. All the parameters agree very well within the experimental error. In the fit parameters for the sample with low anisotropy (sample I), the $z$ component of the bulk magnetization seems to be slightly lower than in sample II (as expected), but the difference might not be significant. $M_{z b}$ is 0.8 in sample I as opposed to 1.0 in sample II; all structural parameters (including the depth of the closure domains) are in close agreement for both samples.

The results indicate that about half the moments in the closure layer lie along the $z$ direction (parallel to the bulk), with the remainder aligned in plane along the $x$ direction. The transition between bulk and closure layer appears to be gradual, with a full width at half maximum of the Gaussian broadening of $20 \AA$, while the top and bottom interfaces of the FePd layer have a magnetic roughness of about $9 \AA$. Preliminary results obtained by fitting reflectivity data suggest that the structural (as opposed to magnetic) roughness of the $\mathrm{FePd} / \mathrm{Pd}$ interfaces is considerably lower at less than $5 \AA$. This difference between structural and magnetic roughness probably reflects to some degree the simplifications in the model for the magnetization, but may also partly be due to a small induced magnetization of those Pd atoms in the capping and buffer layers that are closest to the FePd layer. ${ }^{3}$

\section{CONCLUSIONS}

We studied a series of FePd thin-film samples with low, medium, and high PMA using soft x-ray resonant magnetic scattering at the $\mathrm{Fe}$ and $\mathrm{Pd} L_{3}$ edges. The information from magnetic diffraction rod scans and the x-ray dichroic asymmetry ratio allows us to study the influence of the PMA on the formation of closure domains and to construct a detailed picture of the magnetic structure of the thin films. We find closure domains in the two samples with low and medium PMA, while no closure domains are present in the high-PMA sample. This indicates that closure domains with an in-plane magnetization become energetically unfavorable when a strong PMA (1.6 for sample III) is present. In the two samples with lower PMA data analysis shows the presence of closure domains with an in-plane magnetization to a depth 
of $85 \AA$ in a $420 \AA$ thick film, with a gradual transition to a bulk layer which contains only the PMA domains. The comparative ease with which closure domains can be detected with resonant magnetic scattering clearly demonstrates the potential of the method.

\section{ACKNOWLEDGMENTS}

We would like to thank N. B. Brookes, A. Rogalev, and K. Larsson for their support at the ESRF, and I. Kirkman for his support at the Daresbury SRS.
*Present address: ESRF Grenoble, BP 220, F-38043 Grenoble Cedex, France.

†Present address: Forschungszentrum Jülich, D-5170 Jülich, Germany.

${ }^{1}$ C. Haginoya, K. Koike, Y. Hirayama, J. Yamamoto, M. Ishibashi, O. Kitakami, and Y. Shimada, Appl. Phys. Lett. 75, 3159 (1999).

${ }^{2}$ V. Gehanno, A. Marty, B. Gilles, and Y. Samson, Phys. Rev. B 55, 12552 (1997).

${ }^{3}$ P. Kamp, A. Marty, R. Hoffmann, S. Marchesini, M. Belakhovsky, C. Boeglin, H. A. Dürr, S. S. Dhesi, G. van der Laan, and A. Rogalev, Phys. Rev. B 59, 1105 (1999).

${ }^{4}$ R. Hoffmann, Y. Samson, A. Marty, V. Gehanno, B. Gilles, and J. E. Mazille, J. Magn. Magn. Mater. 192, 409 (1999).

${ }^{5}$ I. Galanakis, M. Alouani, H. Dreyssè, and J. M. Wills, Phys. Rev. B 61, 599 (2000).

${ }^{6}$ C. Kittel, Phys. Rev. 70, 965 (1946).
${ }^{7}$ H. A. Dürr, E. Dudzik, S. S. Dhesi, J. B. Goedkoop, G. van der Laan, M. Belakhovsky, C. Mocuta, A. Marty, and Y. Samson, Science 284, 2166 (1999).

${ }^{8}$ C. Kao, J. B. Hastings, E. D. Johnson, D. P. Siddons, and G. C. Smith, Phys. Rev. Lett. 65, 373 (1990).

${ }^{9}$ L. Sève, J. M. Tonnerre, D. Raoux, J. F. Bobo, M. Piecuch, M. De Santis, P. Troussel, J. M. Brot, V. Chakarian, C. C. Kao, and C. T. Chen, J. Magn. Magn. Mater. 148, 68 (1995).

${ }^{10}$ G. van der Laan, H. A. Dürr, E. Dudzik, M. D. Roper, S. P. Collins, T. P. A. Hase, and I. Pape, Synchrotron Radiat. News 12(3), 5 (1999).

${ }^{11}$ J. P. Hannon, G. T. Trammell, M. Blume, and D. Gibbs, Phys. Rev. Lett. 61, 1245 (1988).

${ }^{12}$ D. B. Pengra, N. B. Thoft, M. Wulff, R. Feidenhansl, and J. Bohr, J. Phys.: Condens. Matter 6, 2409 (1994).

${ }^{13}$ J. P. Hill and D. F. McMorrow, Acta Crystallogr., Sect. A: Found. Crystallogr. 52, 236 (1996). 